\title{
A comparative and combined study of EMIS and GPR detectors by the use of Independent Component Analysis
}

\author{
Axel Morgenstjerne ${ }^{a}$, Brian Karlsen ${ }^{b}$, Jan Larsen ${ }^{c}$, Helge B.D. Sørensen ${ }^{b}$, \\ and Kaj B. Jakobsen ${ }^{b}$ \\ ${ }^{a}$ Danish Defence Research Establishment \\ Ryvangs Allé 1, DK-2100 København Ø, Denmark \\ ${ }^{b} \emptyset$ rsted•DTU, Technical University of Denmark \\ Ørsteds Plads, Building 348, DK-2800 Kongens Lyngby, Denmark \\ ${ }^{c}$ Informatics and Mathematical Modelling, Technical University of Denmark \\ Richard Petersens Plads, Building 321, DK-2800 Kongens Lyngby, Denmark
}

\begin{abstract}
Independent Component Analysis (ICA) is applied to classify unexploded ordnance (UXO) on laboratory UXO test-field data, acquired by stand-off detection. The data are acquired by an Electromagnetic Induction Spectroscopy (EMIS) metal detector and a ground penetrating radar (GPR) detector. The metal detector is a GEM-3, which is a monostatic sensor measuring the response of the environment on a multi-frequency constant wave excitation field $(300 \mathrm{~Hz}$ to $25 \mathrm{kHz})$, and the GPR detector is a stepped-frequency GPR with a monostatic bow-tie antenna $(500 \mathrm{MHz}$ to $2.5 \mathrm{GHz})$. For both sensors the in-phase and the quadrature responses are measured at each frequency. The test field is a box of soil where a wide range of UXOs are placed at selected positions. The position and movement of both of the detectors are controlled by a 2D-scanner. Thus the data are acquired at well-defined measurement points. The data are processed by the use of statistical signal processing based on ICA. An unsupervised method based on ICA to detect, discriminate, and classify the UXOs from clutter is suggested. The approach is studied on GPR and EMIS data, separately and compared. The potential is an improved ability: to detect the UXOs, to evaluate the related characteristics, and to reduce the number of false alarms from harmless objects and clutter.
\end{abstract}

Keywords: Independent component analysis. Unsupervised detection, discrimination, and classification. GPR and EMIS detection of UXOs and landmines.

\section{INTRODUCTION}

Most UXOs and landmines contain metallic parts, and therefore metal detectors of different types have a long history as a tool for location of landmines. However, landmines without metallic parts exists, and those can not be located by traditional metal detection. Detection of non-metallic landmines using Ground Penetrating Radar (GPR) has shown promising results in recent years. Nevertheless, even though the detection of low- and nonmetallic landmines is possible in suitable environments using EMIS and GPR, the detection has proved still to be a hard problem in general. However, the challenge is not to detect the landmine, as long as the signal-to-noise ratio (SNR) for the detector is large enough, but to discriminate landmine signals from clutter signals.

In an UXO or landmine contaminated area, for instance in a previous battle field, there will be found many metallic items that are harmless, which shows the same kind of signal signatures as low-metallic landmines. Furthermore, natural occurrences, like magnetic and non-magnetic stones, roots, and the ground surface, create clutter with high energy which obscure the signals from the low- and non-metallic landmines, and thus gives a low signal-to-clutter ratio (SCR). A low SCR gives a high false alarm rate in the search for landmines and UXO.

Further author information on: $A M$ : axm@oersted.dtu.dk, www.ddre.dk; $B K$ : brk@oersted.dtu.dk, www.oersted.dtu.dk; JL: jl@imm.dtu.dk, www.imm.dtu.dk/ jl; HBDS: hbs@oersted.dtu.dk, www.oersted.dtu.dk; $K B J$ : kbj@oersted.dtu.dk, www.oersted.dtu.dk 
As each alarm must be treated as a true alarm until the cause of the alarm is understood, and any thread is neutralized or security otherwise is established, the true and false alarms requires the same resources. As there can be 1000's of false alarms for each true, it is of great importance to reduce the false alarm rate. As such, the SCR must be enhanced. For that reason, clutter reduction approaches for both EMIS and GPR detectors has been in great interest in the last decade of landmine detection research. Several methods for clutter reduction and detection in GPR and EMIS data exist. The literature suggests a number of methods, such as likelihood ratio testing, ${ }^{1}$ parametric system identification and recognition, ${ }^{2-5}$ wavelet packet decomposition, ${ }^{6,7}$ subspace techniques, ${ }^{8}$ and simple mean subtraction. ${ }^{9-12}$ Several of these methods are based on supervised strategies. That is, models of clutter and targets based on physical parameters or learning from reference signatures or training sets are used to enhance the landmine signatures or recognition. However, because of the statistical nature of the clutter and targets, e.g., if the ground surface is not perfectly flat, the soil and target characteristics changes over position etc., it can be difficult to get robust models. Consequently, if the models are not robust for changes, the detection performance decrease due to classification of clutter as targets or the adverse. The problem of robust models may be solved by the use of unsupervised learning and decomposition methods such as Principal Component Analysis (PCA) or Independent Component Analysis (ICA). We have suggested a promising approach ${ }^{10,11}$ based on decomposition of the GPR signals into clutter and landmine signal subspaces based on PCA and ICA. From visual inspection of the PCA and ICA subspaces, it was shown that a selection of subspaces with target signatures are able to recover landmine signals and suppress clutter signals. In this paper, we extend this work by studies of unsupervised detectors based on ICA. The idea is to perform the detection without visual inspection of the ICA subspaces.

We have measured different UXO targets in a laboratory setup with an artificial UXO-field. The data were acquired using a stepped-frequency GPR and using a continuous wave EMIS. Afterwards, the measurements have been analyzed using the suggested ICA based detection approach which is discussed in section 2. The acquired data sets are presented in section 3. And finally, the results and the conclusion are presented in section 4 and 5 .

\section{UNSUPERVISED LANDMINE DETECTION BASED ON ICA}

In a GPR and EMIS system several factors contributes to the difficulty in the design of a reliable detector. The most distinctive factor is the high level of ground clutter in the data, which introduces a low SCR. Consequently, a low SCR gives an unreliable detection. In addition to the low SCR we also have the problem that the ground clutter and in particular the target signatures changes, as a function of the surrounding environment, such as the soil type and the humidity in the soil. Therefore, to design a reliable "all-around" detector depending on ground clutter and target signatures would possibly require examples of signatures for not only every landmine type, but also every landmine type on every site, which in practice is improbable.

A solution to this problem is to design a detector which we are able to "train" from on-site ground clutter and target signature examples. However, examples of target signatures may still be impossible to get, because in several cases we do not know what the target is. This bring us to the solution of designing a detector depending on ground clutter signatures only, which at most is easiest to collect. The assumption for such kind of a detector is to find features in the ground clutter signatures that capture the statistical nature of the ground clutter. Thus a possible detection approach is to compare features found from new measurement with the "trained" ground clutter features. New measurements that show significant distinct features are then considered to contain targets. Hence, a clutter-target discrimination and detection in the GPR and EMIS sensor data can be performed by finding prominent features that discriminates the data best.

In general several types of deterministic or statistical features can be applied for this approach. We apply statistical features found by exploiting the redundancy in the data by the use of ICA. This approach is very similar to the approach given in S-H. Yu et al. ${ }^{13}$, which is based on PCA. Redundancy may hide useful information in the data, which can be used to find prominent features. Redundancy arises when one collects multivariate data because of correlation between variables. E.g., in a GPR or EMIS system the sensor data is measured in a arbitrary grid as function of frequency or range at each grid position. This repetition of the measurements at each grid position induces a high redundancy in the data. However, high-dimensional representation of such data does not allow easy exploitation of the redundancy, it is of great interest to find prominent representations 
of the data that obtain simplicity for better understanding, visualization and interpretation of the data and the redundancy.

An important unsupervised statistical method in this context, is PCA. PCA of high-dimensional multivariate data is based on a linear transformation which project the data unto orthogonal subspaces of decreasing variance, the principal components. Thus, by PCA we are able to exploit the correlation in the data. In the literature it has been argued, that natural structured data is not orthogonal but independent, which suggest ICA as a natural choice for finding prominent subspaces in data. In the following we present a detector based on ICA which is able to detect distinct variations from the mean in the data, which is considered to be target signatures.

\subsection{Independent Component Analysis}

Given only sensor observations that are assumed to be an unknown linear mixture of unobserved, statistically independent components, the problem of ICA is to recover the independent components from the observed data alone. This goal can be meet by linear transformation of the sensor observations, such that the resulting outputs from the transformed sensor observations are as statistical independent from each other as possible. When compared to PCA, which is a correlation based transformation, ICA not only decorrelates the sensor observation of the linear mixed components, but also reduces the higher-order statistical dependencies between them.

Consider now a zero-mean $M$-dimensional random vector, $\mathbf{s}=\left[s_{1}, s_{2}, \ldots, s_{M}\right]^{\top}$, corresponding to $M$ realvalued, independent components $s_{i}$. The probability density function (pdf) of the vector $\mathbf{s}$ can be written as the product of the marginal distributions of $s_{i}$,

$$
p(\mathbf{s})=\prod_{i=1}^{M} p\left(s_{i}\right) .
$$

To fulfill the requirements of statistical independence, the pdf product equality in eqr. (1) must be satisfied. Assuming now the sensor observations given by the real-valued $N$-dimensional zero-mean random vector $\mathbf{x}=$ $\left[x_{1}, x_{2}, \ldots, x_{N}\right]^{\top}$, corresponding to $N$ observed signals, which are linear combinations of the $M$ independent components s. As such, a linear mixture model is defined as,

$$
\mathbf{x}_{t}=\mathbf{A} \mathbf{s}_{t},
$$

where $\mathbf{A}$ is a real-valued full rank $N \times M$ scalar mixing matrix. The index $t$ holds for the sample vector number* A consequence of the mixing process is that the pdf product equality of the observed signals is not satisfied, i.e.,

$$
p(\mathbf{x}) \neq \prod_{i=1}^{N} p\left(x_{i}\right) .
$$

The goal of ICA is to recover the independent components, $\mathbf{s}$, from the dependent sensor observations, $\mathbf{x}$, without any information about mixing matrix, A. This can be done by applying a linear transformation system (or linear separation) on $\mathbf{x}$ expressed written as

$$
\hat{\mathbf{s}}=\mathbf{W} \mathbf{x}=\mathbf{W A s}
$$

Thus, the ICA solution for this problem is to find a linear transformation matrix $\mathbf{W}$, of dimension $M \times N$, which makes the outputs $\hat{\mathbf{s}}=\left[\hat{s}_{1}, \hat{s}_{2}, \ldots, \hat{s}_{M}\right]^{\top}$ from the linear transformation of the dependent sensor signals $\mathbf{x}$ as independent as possible. The transformation $\hat{\mathbf{s}}$ is an estimate on $\mathbf{s}$, and if we for simplicity have, that $N=M$ and $\mathbf{W}=\mathbf{A}^{-1}$, then the independent components are exactly recovered up to permutation and scale. Hence, $\hat{\mathbf{s}}=\mathbf{A}^{-1} \mathbf{A} \mathbf{s}=\mathbf{I s}$, where $\mathbf{I}$ is an identity matrix.

In the application of GPR and EMIS, the independent components, can be considered as hidden source signals in the sensor data, which through a mixture model generates the observed data. Thus, under the hidden source assumption, recovering these sources will provide us with useful information about how the data is generated.

\footnotetext{
${ }^{*}$ Usually we have $T$ samples of the observation vector, i.e., the GPR and EMIS data are collected from a large grid. Therefore also $T$ samples of the independent component vector.
} 
In the GPR and EMIS sensor data we will have a greater number of observations than sources. Furthermore we may have badly scaled data with additive noise. This give rise to noisy and ill-conditioned problems for ICA estimation. Pre-whitening of the GPR and EMIS data is optional in order to improve convergence speed for ill-conditioned problems. We provide PCA which is a orthogonal transform and decorrelation approach for pre-whitening of the GPR and EMIS data. ${ }^{\dagger}$ PCA can be executed using singular value decomposition (SVD) written as

$$
\mathbf{x}=\mathbf{U D V}^{\top}=\sum_{i=1}^{K} \mathbf{u}_{i} \mathbf{D}_{i, i} \mathbf{v}_{i}^{\top}
$$

Here, the $N \times K$ matrix $\mathbf{U}=\left[\mathbf{u}_{1}, \mathbf{u}_{2}, \ldots, \mathbf{u}_{K}\right]$ and the $T \times K$ matrix $\mathbf{V}=\left[\mathbf{v}_{1}, \mathbf{v}_{2}, \ldots, \mathbf{v}_{K}\right]$ represent the orthonormal basis vectors, i.e., eigenvectors of the symmetric matrices $\mathbf{y y}^{\top}$ and $\mathbf{y}^{\top} \mathbf{y}$, respectively. $\mathbf{D}$ is a $K \times K$ diagonal matrix of singular values ranked in decreasing order, thus $D_{i-1, i-1}>D_{i, i}, \forall i \in[2 ; K]$. The SVD identifies a set of uncorrelated components, the principal components (PC) given by: $\mathbf{y}_{i}=D_{i, i} \mathbf{v}_{i}$, enumerated by the component index $i=1,2, \ldots, T$ and $\mathbf{y}_{i}=\left[y_{i}(1), \cdots, y_{i}(T)\right]^{\top}$. The dimension of the PCA data set will be $K \leq N$. That is, we model $\mathbf{x}$ only from non-zero eigenvalues and further have the possibility of projecting onto a subspace. Pre-whitening and subspace projection of $\mathbf{x}$ is obtained by

$$
\widetilde{\mathbf{x}}=\widetilde{\mathbf{U}}^{\top} \mathbf{x},
$$

where $\widetilde{\mathbf{U}}=\left[\mathbf{u}_{1}, \mathbf{u}_{2}, \cdots, \mathbf{u}_{M}\right]$ is $N \times M$ and $\widetilde{\mathbf{x}}$ is a $M \times T$ matrix. Hence, after pre-whitening and projection the $K \times K$ ICA problem with mixing matrix $\boldsymbol{\Phi}$ is

$$
\widetilde{\mathbf{X}}=\mathbf{\Phi S} \quad, \quad \widehat{\mathbf{S}}=\mathbf{W X}=\mathbf{\Phi}^{-1} \widetilde{\mathbf{U}}^{\top} \mathbf{X}
$$

In the application of landmine detection, the ICA can be used to detect landmines and reduce clutter. By selecting components which mainly carry mine information, say $\widehat{\boldsymbol{s}}_{k}$, we can remove clutter. The reconstructed signal space in the original GPR signal space is then

$$
\widehat{\mathbf{x}}_{k}=\widehat{\mathbf{U}} \boldsymbol{\Phi} \widehat{\mathbf{s}}_{k}
$$

There exist a wide range of algorithms which solves for the ICA problem. Among the most used algorithms we find algorithms based on entropy minimization, minimization of mutual information, optimization of a nongaussianity measure, and maximum likelihood (see Lee ${ }^{14}$ ). The method we employ for on the EMIS and GPR data is the extended information maximization (extended infomax) approach ${ }^{15}$, which is an iterative learning algorithm. The optimization criterion for this algorithm is minimization of mutual information between the variables in $\hat{\mathbf{s}}$.

\subsection{An unsupervised landmine detection method}

The amplitude of the ground clutter can be characterized by statistical fluctuations which can be described in terms of a pdf. E.g., for a GPR the total echo from the ground surface can be described by a superposition of several individual scatters (the so called facets model). ${ }^{16}$ If we consider that the magnitude of the echo from each of all the individual scatters are independent, then the amplitude of the ground clutter is described by a gaussian pdf, if the ground surface is located in the far-field of the antenna. ${ }^{16}$ However, in this paper we consider that the in-phase and quadrature clutter in the multivariate GPR and EMIS sensor observations can be describe the by the multivariate gaussian pdf given by the mean $\mathbf{m}_{c l}$, and the covariance matrix $\mathbf{K}_{c l}$ :

$$
p_{c l}\left(\mathbf{x} \mid \mathbf{m}_{\mathbf{c l}}, \mathbf{K}_{\mathbf{c l}}\right)=\frac{1}{\sqrt{\operatorname{det}\left(2 \pi \mathbf{K}_{c l}\right)}} \exp \left[-\frac{1}{2}\left(\mathbf{x}_{c l}-\mathbf{m}_{c l}\right)^{\top} \mathbf{K}_{c l}^{-1}\left(\mathbf{x}_{c l}-\mathbf{m}_{c l}\right)\right] .
$$

where the mean is obtained by

$$
m_{c l, i}=\frac{1}{T} \sum_{i=1}^{T} x_{t, i} .
$$

\footnotetext{
${ }^{\dagger}$ If $\mathbf{A}$ is non-square ill-conditioned problems may arise.
} 
An estimate of covariance matrix can be found from the eigenvectors or the ICA basis vectors as

$$
\hat{\mathbf{K}}_{c l}=\tilde{\mathbf{U}} \mathbf{D} \widetilde{\mathbf{U}}^{\top}, \quad \hat{\mathbf{K}}_{c l}^{-1}=\widetilde{\mathbf{U}} \mathbf{D}^{-1} \widetilde{\mathbf{U}}^{\top},
$$

and

$$
\hat{\mathbf{K}}_{c l}=\sum_{i=1}^{M} \mathbf{U} \mathbf{D}^{\frac{1}{2}} \mathbf{a}_{i} \mathbf{a}_{i}^{\top} \mathbf{D}^{\frac{1}{2}} \mathbf{U}^{\top} \quad \text { and } \quad \hat{\mathbf{K}}_{c l}^{-1}=\sum_{i=1}^{M} \mathbf{U} \mathbf{D}^{-\frac{1}{2}} \mathbf{w}_{i}^{\top} \mathbf{w}_{i} \mathbf{D}^{-\frac{1}{2}} \mathbf{U}^{\top},
$$

respectively. The unsupervised landmine detection approach is based on testing how far from the ground clutter model in equ. 9 one new observation is. From the clutter model in equ. 15 we create a likelihood detector from which we can measure the distinct difference between the model and the observation. We define a likelihood by

$$
\ln \left(p_{c l}\right)(\mathbf{x})=-\frac{N}{2} \ln (2 \pi)-\frac{1}{2} \ln \left(\operatorname{det} \mathbf{K}_{c l}\right)-\frac{1}{2}\left(\mathbf{x}-\mathbf{m}_{x}\right)^{\top} \mathbf{K}_{c l}^{-1}\left(\mathbf{x}-\mathbf{x}_{x}\right),
$$

From the estimate of the covariance matrix, given by $\hat{\mathbf{K}}$ spanning the subspace of $M$ selected independent components we get the following detector,

$$
-\frac{1}{2}\left(\mathbf{x}_{t}-\mathbf{m}_{c l}\right)^{\top} \hat{\mathbf{K}}_{c l}^{-1}\left(\mathbf{x}_{t}-\mathbf{m}_{c l}\right)_{>}^{\leq} \eta_{c l},
$$

where $\eta_{c l}$ is a threshold. Detection values greater than or equal to the threshold is assumed to be from targets, whereas values smaller than the threshold is assumed to be from clutter. First, we divided the data into training set and test set. Using the training set, we built a model to effectively describe the target data using a variant of the well-known Principal Component Analysis (PCA), which under gaussian noise assumptions, provides the optimal estimate (in the maximum likelihood sense) of the subset that best describing the data. 


\section{THE GPR AND EMIS DATA SETS}

The data for the test of the ICA detection process were acquired using GPR and EMIS sensor. The GPR we are employing is an stepped-frequency GPR with a wide-band monostatic bow-tie antenna. The in-phase $\left(\mathrm{I}_{g p r}\right)$ and quadrature $\left(\mathrm{Q}_{g p r}\right)$ of the reflections are acquired at 201 frequencies equally space in the range from $500 \mathrm{MHz}-2.5 \mathrm{GHz}$. The GPR is operating $5 \mathrm{~cm}$ above the ground surface. The principle in the stepped-frequency GPR is that the in-phase $\left(\mathrm{I}_{g p r}\right)$ and quadrature $\left(\mathrm{Q}_{g p r}\right)$ of the reflected field from the scatters from the ground surface and from under the surface is measured at different frequencies. The scattered field is generated from electromagnetic changes in the medium where the radiated field propagates. The electromagnetic induction

The EMIS Sensor

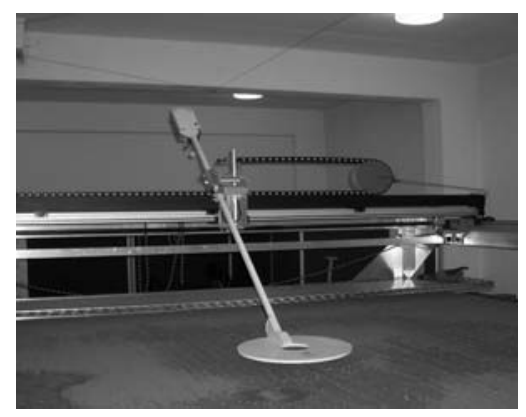

The GPR Sensor

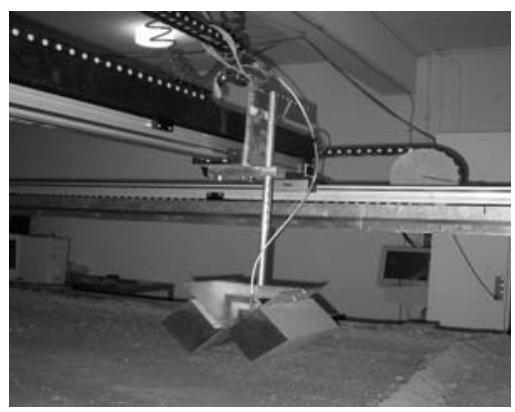

The Test-field

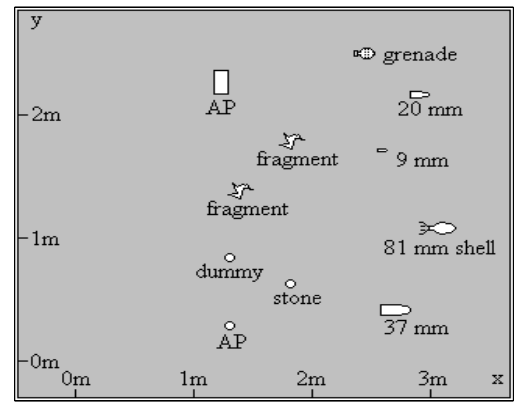

Figure 1. The artificial UXO- and landmine-field, and sensor setup. Picture to the left: The GEM-3 EMIS sensor mounted in the 2D-scanner. Picture in the middle: The bow-tie GPR antenna mounted in the 2D-scanner. Sketch to the right: The test-field setup.

spectroscopy ${ }^{17,18}$ instrument we are using is the GEM-3 ${ }^{19}{ }^{19}$ a continues wave active detector that we have set up to measure in-phase and quadrature components of the response of the surroundings to the excitating magnetic field with 5 frequency components. The principle is, that GEM-3 measures the secondary field, generated by the environment as a response to the primary field. Due to the geometry of the GEM-3 coils, this means that magnetic items, that induce deformations of the primary field, will give rise to signals that are in phase with the primary field, while induced currents in conducting items will give rise to signals that are out of phase with the primary field. Thus, one will expect items with magnetic properties to appear in the in-phase measurements while items with conducting properties will appear in the quadrature measurements. This is seen particulary at low frequencies. ${ }^{17}$ Of course, as all real life objects has both magnetic and conducting properties and as these depends on the frequency due to many parameters, this will result in different spectra for different items, not only depending on the item itself (material, size and shape) but also on mutual orientation and position (distance, depth) and surrounding environment. It has been suggested, ${ }^{20}$ that identification of mines and other items could be possible from EMIS spectra. This seems possible for different mines measured in free air, but when small mines are measured in soil, and background signals and clutter contributes to the spectra, identification turns out to be very difficult or even impossible.

As mentioned, in our setup of the GEM-3, we acquire 10 measurements at each sample point. The measurements are the in-phase and the quadrature relative component at 5 frequencies, and the frequencies we have selected are $325 \mathrm{~Hz}, 925 \mathrm{~Hz}, 2775 \mathrm{~Hz}, 8175 \mathrm{~Hz}$, and $20025 \mathrm{~Hz}$. The frequencies are distributed almost logarithmic periodically and selected so that they are not multiplies of $50 \mathrm{~Hz}$ (the power line frequency). The primary coil of the GEM-3 generates an alternating magnetic field with the 5 frequency components. Due to the geometry of the primary coil, which consists of 2 opposite coils, the primary magnetic field is configured almost as a dipole, but is balanced out at the secondary coil. As the primary field is suppressed at the secondary coil, the response of the environment can be measured as the induced current in the secondary coil. For each frequency, the in-phase and quadrature component in the current of the secondary coil are measured relative to the current in the primary coil and expressed in ppm.

During the measurements with the GEM-3, the scanner moves the instrument in a pattern of lines from side to side. The movement is continuous along the line with acceleration when it starts and deceleration at the 
end of the line. Then the scanner progress to the next line, with a spacing of $20 \mathrm{~mm}$ between the lines, and the instrument is moved along the next line in the opposite direction. The computer records the measurements from the GEM-3 with time-tags and it records the scanner position with time-tags. From these recordings a data file is generated with positions (corrected for instrument offset) and measurements. The $20 \mathrm{~mm}$ spacing between lines was due to practical reasons, and in the analysis we interpolated to a $10 \mathrm{~mm}$ by $10 \mathrm{~mm}$ grid to match the data sampled by GPR. The interpolation did not affect the results. During the measurements with the GPR, the scanner moves in a similar pattern, that is lines separated by $10 \mathrm{~mm}$. But along the line, the scanner stops for each GPR measurement at $10 \mathrm{~mm}$ intervals, and the measurement is carried out at a steady position. The scanner is controlled from the computer, in a way that the computer is coordinating the experiment by positioning and moving the instruments, and acquiring the data from the instruments.

Different targets of UXO and landmines are buried in the soil. A plastic AP-mine, an rubber AP-mine dummy, a stone, fragments of a shell, an AP-mine with metal trigger, a $37 \mathrm{~mm}$ projectile, a $81 \mathrm{~mm}$ shell, a 9 $\mathrm{mm}$ projectile, a $20 \mathrm{~mm}$ projectile, and a grenade. All targets was placed flush with the surface. In figure $1 \mathrm{a}$ sketch of the setup is shown.

\section{RESULTS ON EXPERIMENTS}

The GPR and EMIS sensor data described in section 3 was used to test the suggested ICA detection process described in subsection 2.2. The collected data from the two sensors was arranged separately in two similar sensor observation matrices. The structure of the sensor observation matrices is given by

$$
\mathbf{x}=\left[\begin{array}{cccc}
I_{1}\left(f_{1}\right) & I_{2}\left(f_{1}\right) & \cdots & I_{T}\left(f_{1}\right) \\
I_{1}\left(f_{2}\right) & I_{2}\left(f_{2}\right) & \cdots & I_{T}\left(f_{2}\right) \\
\vdots & \vdots & \ddots & \vdots \\
I_{1}\left(f_{n}\right) & I_{2}\left(f_{n}\right) & \cdots & I_{T}\left(f_{n}\right) \\
Q_{1}\left(f_{1}\right) & Q_{2}\left(f_{1}\right) & \cdots & Q_{T}\left(f_{1}\right) \\
Q_{1}\left(f_{2}\right) & Q_{2}\left(f_{2}\right) & \cdots & Q_{T}\left(f_{2}\right) \\
\vdots & \vdots & \ddots & \vdots \\
Q_{1}\left(f_{n}\right) & Q_{2}\left(f_{n}\right) & \cdots & Q_{T}\left(f_{n}\right)
\end{array}\right]
$$

The columns of $\mathbf{x}$ are the sensor data measured at one sensor position, where the first $n$ values is the in-phase data and the last $n$ values are the quadrature data. As such, for the GPR data $\mathbf{x}$ has the dimension $402 \times T$, where $T$ is number of sensor positions, or observation examples. Similarly, for the EMIS's data $\mathbf{x}$ has the dimension $10 \times T$.

From the two sets of $\mathbf{x}$ an area with no targets but clutter only was then selected, and from these data two new clutter observation matrices was created $\mathbf{x}_{c l}$. From each of these matrices the first 10 principal components was selected, which led to a total variance of approx. 99\% of the data. From this subspace the ICA basis vectors $\mathbf{a}_{i}$ was estimated. The 3 ICA basis vector that shows best performance is then selected and the covariance matrix $\hat{\mathbf{K}}_{c l}$ was estimated from eq. 15 .

To compare a ICA clutter model with a ICA target model, similar data set was created from an area with a target. Again the ICA basis vectors was estimated from a subspace spanning the first 10 principal components.

In figure 2 are samples of the IC's $\left(\mathbf{s}_{i}\right)$ and associated ICA basis vectors $\mathbf{a}_{i}$ from the GPR data where only clutter is present shown. In comparison are samples of the IC's and associated ICA basis vectors from the GPR data where both clutter and target is present shown in figure 3. From the IC and ICA basis vectors it is not visually clear what is target and what is clutter.

In figure 4 are samples of the IC's $\left(\mathbf{s}_{i}\right)$ and associated ICA basis vectors $\mathbf{a}_{i}$ from the EMIS data where only clutter is present shown. In comparison are samples of the IC's and associated ICA basis vectors from the EMIS data where both clutter and target is present shown in figure 5. From the IC and ICA basis vectors it is not visually clear what is target and what is clutter.

Both the results for the GPR- and the EMIS-sensor are shown. In the left images the applied model is the clutter model, and in the right it is the target model. In the top row the data, which the model is applied to, is 
IC's from GPR Data with Clutter Only
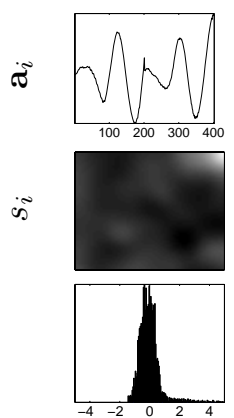
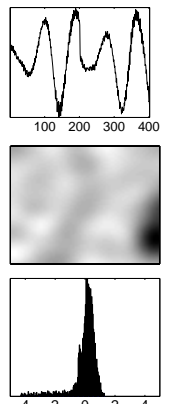
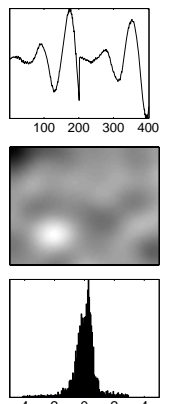
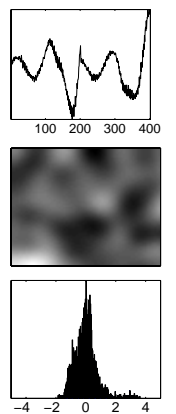
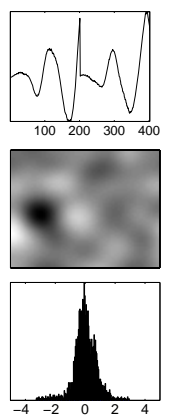
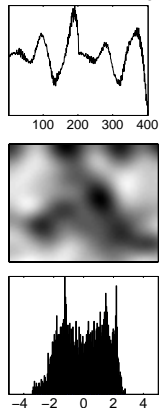
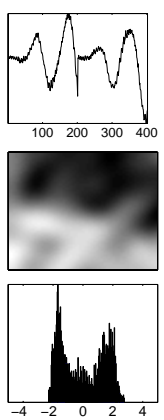
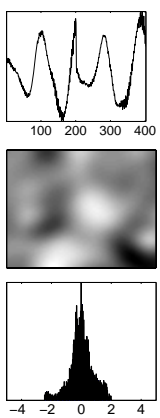

Figure 2. A sample of $8 \mathrm{IC}$ estimated from GPR data collected from a regular area with clutter only, are shown here. Top row: the basis vectors, that shows how the IC contributes to the 402 measurement channels. Middle row: image representations of the IC's. Bottom row: the respective histogram of the IC's.

IC's from GPR Data with Clutter and Target
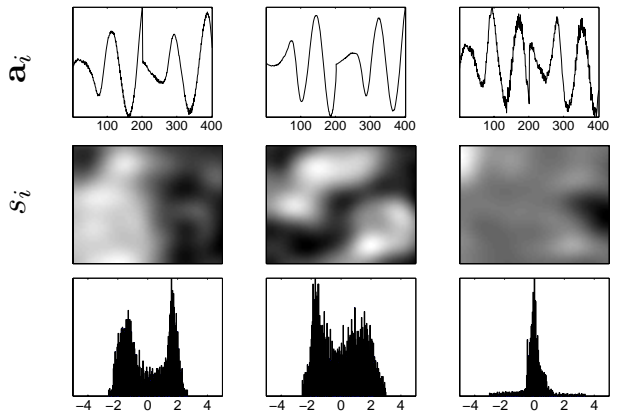
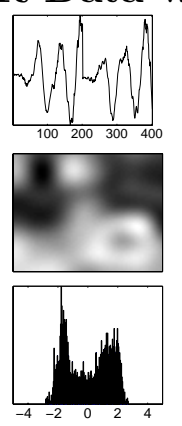
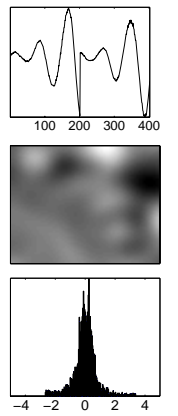
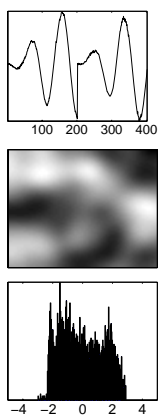
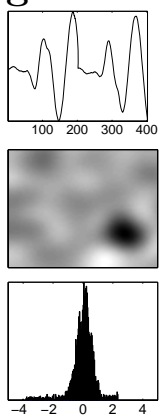
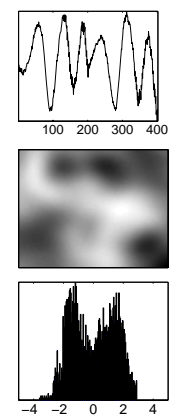

Figure 3. A sample of 8 IC estimated from GPR data collected from a regular area with targets and clutter, are here shown. Top row: the basis vectors, that shows how the IC contributes to the 402 measurement channels. Middle row: image representations of the IC's. Bottom row: the respective histogram of the IC's. In contrast to the clutter measurements, there is a more clear separation of components into a group of subgaussian and a group of supergaussian distributions.

clutter data, and in the bottom it is target data. It is seen, how the target stands out as non-clutter, when the clutter model is applied. Notice the different grey scales on the images.

In figure 8 the results were we apply the suggested selection and detection procedure on the data are shown. In order to evaluate the quality of the detection process we use the measure of correct detected target observations versus detection of clutter observations. The IC's resemble the PC's, which may be due to the fact that the data is only presented by a low number of components. The ROC curves shows that ICA gives a better performance than PCA (see figure 7). However, due to the low dimensionality the PCA and ICA results are very similar. Hence we suggest to increase the dimension of the data.

Before the ICA was applied to the data, PCA was applied, in order to give a feature subset describing most of the variance. In case of the PCA the first 8 to 15 principal components was selected, which led to a total variance of $99 \%$. After training the selection procedure was applied to the estimated subset. 
IC's from EMIS Data with Clutter Only
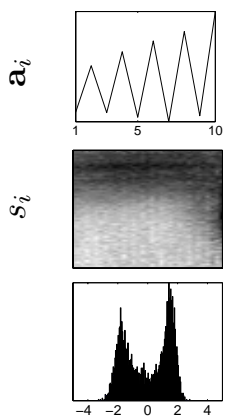
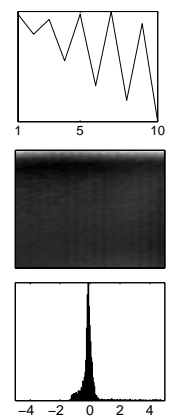
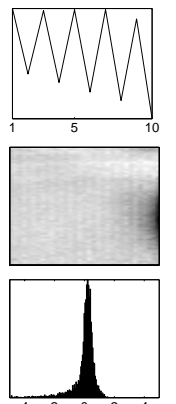
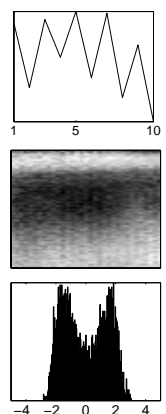
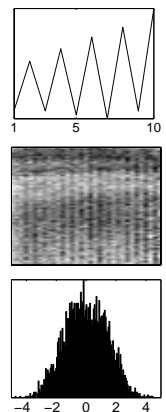
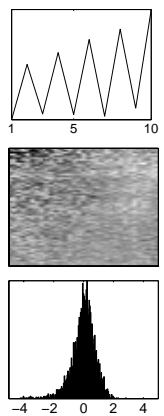
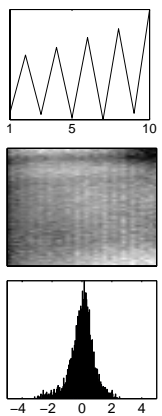
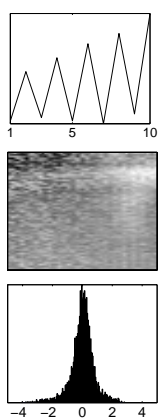

Figure 4. A sample of 8 IC estimated from EMIS data collected from a regular area with clutter only, are here shown. Top row: the basis vectors, that shows how the IC contributes to the 10 sensor measurements. Middle row: image representations of the IC's. Bottom row: the respective histogram of the IC's.

\section{IC's from EMIS Data with Clutter and Target}
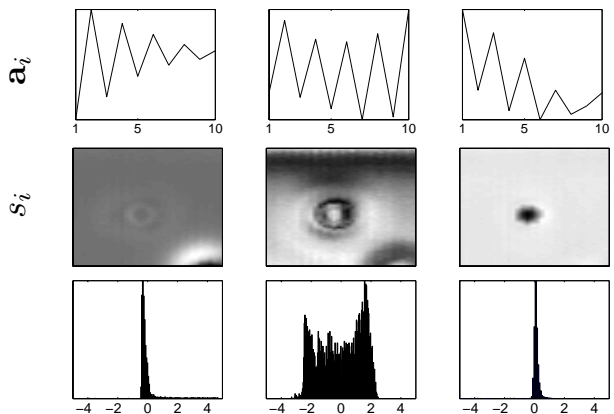
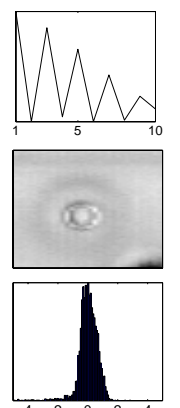
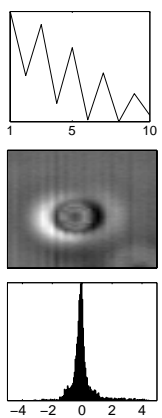
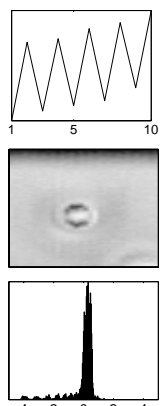
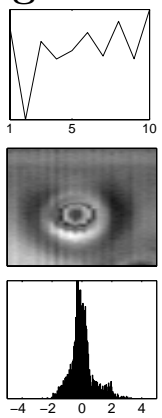
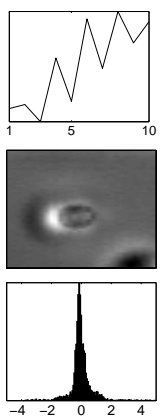

Figure 5. A sample of 8 IC estimated from EMIS data collected from a regular area with clutter and target present, are here shown. Top row: the basis vectors, that shows how the IC contributes to the 10 sensor measurements. Middle row: image representations of the IC's. Bottom row: the respective histogram of the IC's.

\section{CONCLUSION}

An ICA based detection and classification model was employed on GPR and EMIS data, and results were compared. The ICA method is able to identify artifacts such as, e.g., landmines in the data. However, the performance is not significantly better than PCA. This is believed to be due to a lot of unobserved parameters such as size of data set and dimensionality in the data. From known clutter it is possible to create a model to estimate clutter and hence discriminate clutter from target data.

Using estimation based on maximum likelihood we find, that ICA very effectively can be applied to suppress clutter signals and enhance object signals. In this study we have applied ICA for classification of clutter, and we are able to recognize targets as non-clutter objects. However, it seems possible also to classify different objects by selective training on object data. We are planning further experiments to obtain sufficient data for such classification.

Further research in classification elements for characterization, like position, depth, size, shape and material is to be done, and several strategies for the decomposition with respect to the position or frequency, and to the signal representation (complex or amplitude/phase) are to be examined. An obviously next step in this study is to apply the ICA method to the data from EMIS and GPR in fusion. We expect, that the classification thereby will be improved and consolidated. 
GPR

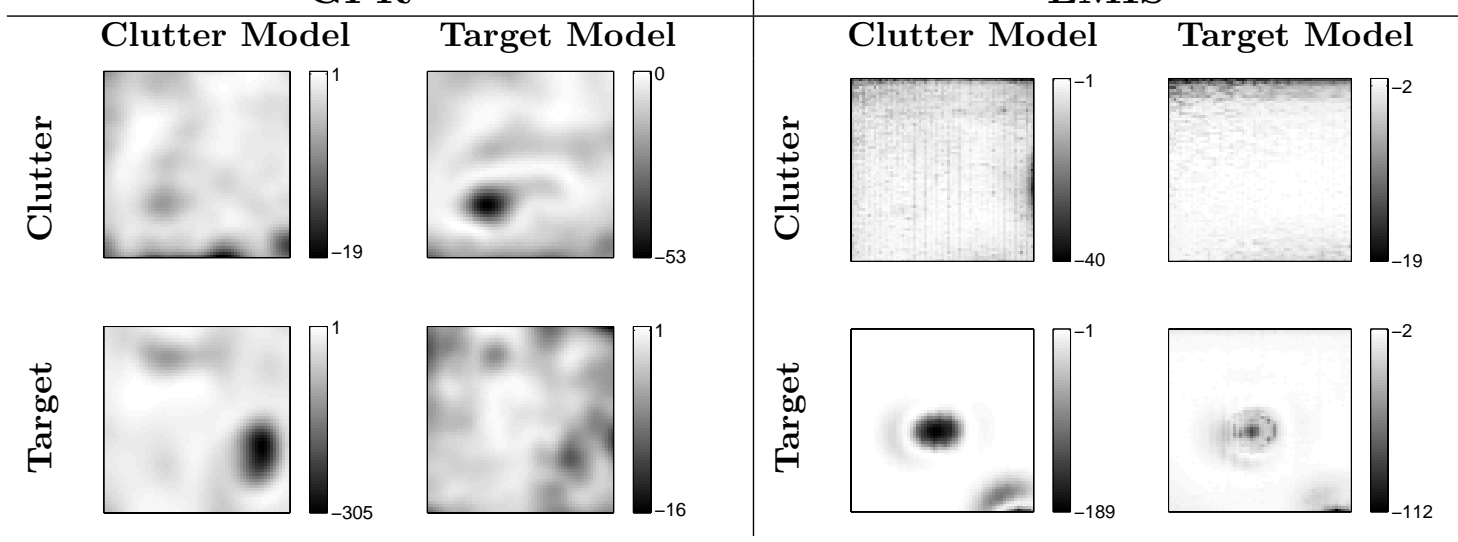

Figure 6. We have tested an ICA clutter model against an ICA target model on data sets from both GPR and EMIS. We have trained the detector given in equ. 14 on clutter and a similar detector is trained on target and clutter. The images shows the detection results. The 4 examples for each sensor shows: Top left: We have trained on clutter and tested on clutter. For both sensors, the resulting match is fine. Bottom left: We have trained on clutter and tested on an area with a target. For both sensors the model describes well the area outside the target, but the target itself stands out, because the clutter model fails to describe it. Thus the target can be classified as non-clutter. Pay attention to the gray scale, that goes to much more negative values in this case, and thus a good contrast is observed. Top right: We have trained on an area with a target and tested on an area with clutter. As there is also some clutter in the target area, the model does actually also describe clutter reasonably well, particulary in the case of the EMIS sensor. Bottom right: We have trained on an area with a target and tested on itself. It is found, that the model describes the target area well. For the EMIS sensor, there is seen some traces of the target as misfits. Still it fits clearly better that the clutter model.
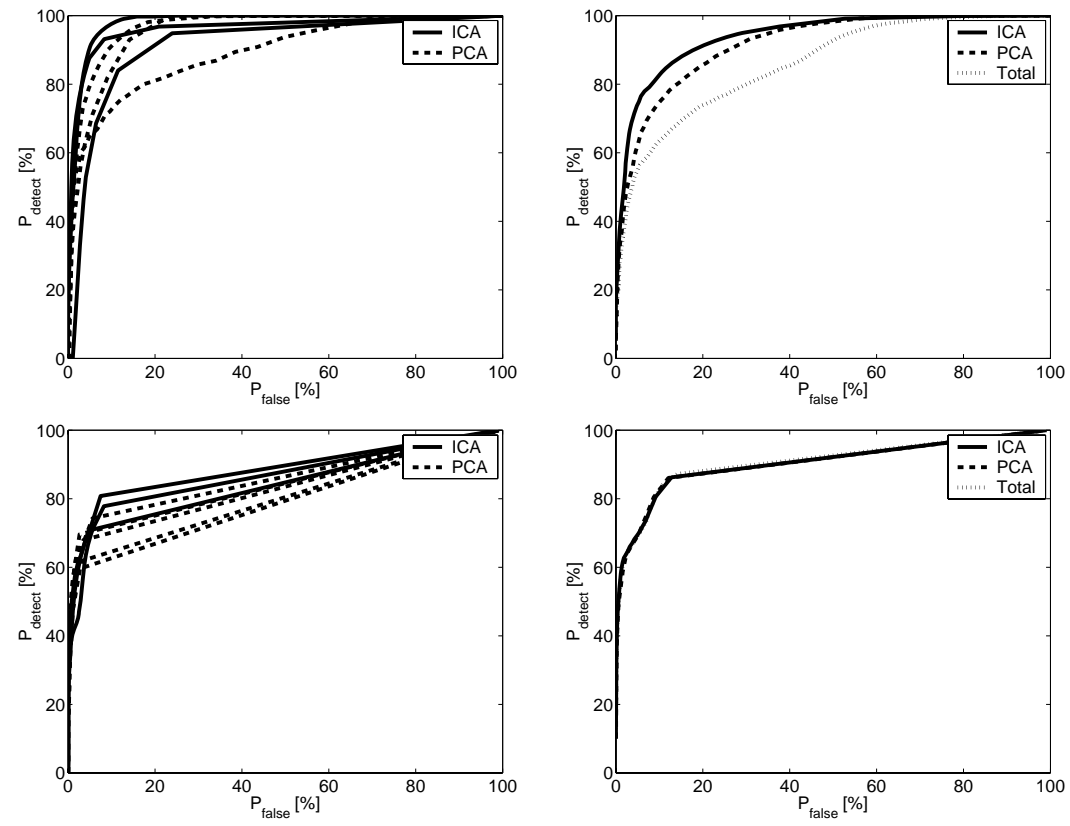

Figure 7. ROC-curves from analyzed GPR-data. Left graph: We have selected the 3 IC's and the 3 PC's giving the best detection and displayed their ROC-curves together. Right graph: Using the subspace defined by the 3 best IC's we display the resulting ROC-curve for ICA, and likewise for PCA. We also display the "Total" ROC-curve using the full data space. It is seen, that we obtain the best detection by the use of ICA. 

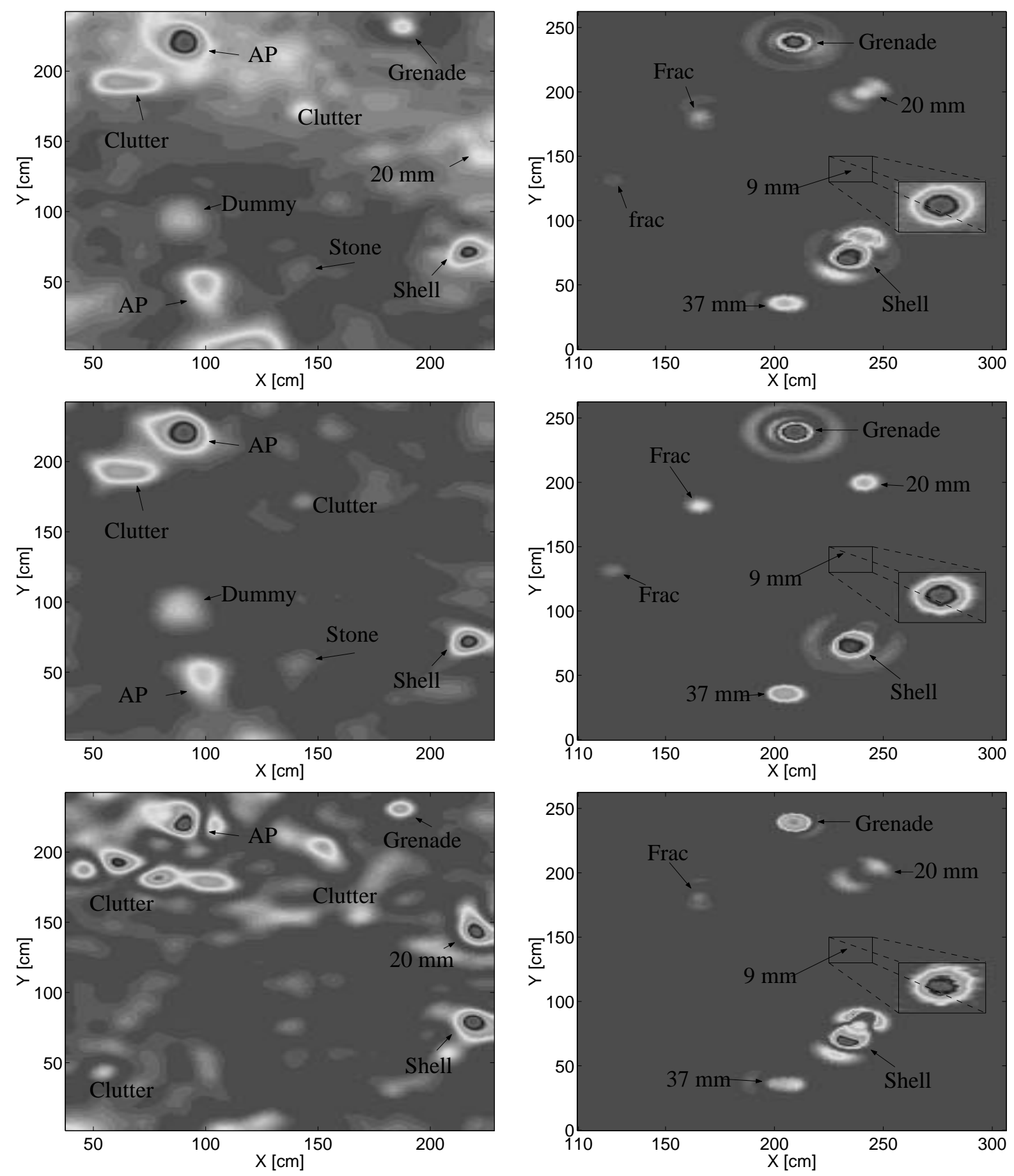

Figure 8. Examples of detection analysis on the GPR-data (to the left) and on the EMIS-data (to the right). The top images shows detection applied to the full data space, while the middle and bottom images shows selected IC's. It is seen, how different objects appears differently in different IC's. Thus, depending on which object one wish to detect, different IC's should be utilized. This correspondence is established by the training of a model on data. For the EMIS-data, there is a clear detection of all metallic objects, and the ICA does not give much improvement of the detection. However, scattered fields from the objects are suppressed in certain IC's, which makes the object appear more clearly and localized. 


\section{REFERENCES}

1. H. Brunzell, "Clutter reduction and object detection in surface penetrating radar," in IEE Radar '97, 449, pp. 688-691, 1997.

2. J. Brooks, L. van Kempen, and H. Sahli, "Primary study in adaptive clutter reduction and buried minelike target enhancement from gpr data," in Det. and Rem. Techn. for Mines and Minelike Targets V, SPIE 4038, pp. 1183-1192, 2000.

3. L. van Kempen, H. Sahli, E. Nyssen, and J. Cornelis, "Signal processing and pattern recognition methods for radar ap mine detection and indentification," Det. of Aband. Land Mines 458, pp. 81-85, 1998.

4. A. van der Merwe and I. Gupta, "A novel signal processing technique for clutter reduction in gpr measurements of small, shallow land mines," IEEE Transactions on Geoscience and Remote Sensing 38, pp. 2627$2637,2000$.

5. J. Salvati, C. Chen, and J. Johnson, "Theoretical study of a surface clutter reduction algorithm," in 1998 IEEE International Geoscience and Remote Sensing, 3, pp. 1460-1462, 1998.

6. D. Carevic, "Clutter reduction and target detection in ground penetrating radar data using wavelets," in Det. and Rem. Tec. for Mines and Minel. Targ. IV, SPIE, ed., 3710, pp. 973-997, 1999.

7. H. Deng and H. Ling, "Clutter reduction for synthetic aperture radar images using adaptive wavelet packet transform," in IEEE Int. Ant. and Propaga. Soc. Symp., 3, pp. 1780-1783, 1999.

8. A. Gynatilaka and B. Baertlein, "A subspace decomposition technique to improve gpr imaging of antipersonnel mines," in Detect. and Rem. Techn. for Mines and Minelike Targets V, SPIE, ed., 4038, pp. 1008$1018,2000$.

9. D. Daniels, Surface Penetrating Radar, IEE, 1996.

10. B. Karlsen, J. Larsen, K. Jakobsen, H. Sørensen, and S. Abrahamson, "Antenna characteristics and airground interface deembedding methods for stepped-frequency ground penetrating radar measurements," in Detect. and Rem. Techn. for Mines and Minelike Targets V, SPIE 4038, pp. 1420-1430, 2000.

11. B. Karlsen, J. Larsen, H. Sørensen, and K. Jakobsen, "Comparison of pca and ica based clutter reduction in gpr systems for anti-personal landmine detection," in 11th IEEE Workshop on Statistical Signal Processing, pp. 146-149, 2001.

12. A. Shaw and V. Bhatnagar, "Automatic target recognition using eigen-templates," in SPIE Conference on Algorithms for Synthetic Aperture Radar Imagery V, 3370, pp. 448-459, 1998.

13. S.-H. Yu, R. K. Mehra, and T. R. Witten, "Automatic mine detection based on ground penetrating radar," in Detection and Remediation Technologies for Mines and Mine-Like Targets IV, A. C. Dubey, J. F. Harvey, J. Broach, and R. E. Duncan, eds., Proc. SPIE 3710, pp. 961-972, 1999.

14. T. Lee, Independent Component Analysis: Theory and Applications, Kluwer Academic Publishers, 1998.

15. T.-W. Lee, M. Girolami, and T. J. Sejnowski, "Independent component analysis using an extended infomax algorithm for mixed aub-gaussian and super-gaussian sources," Neural Computation 11, pp. 417-441, 1999.

16. M. Skolnik, Introduction to Radar Systems, Printice Hall, 1968.

17. I. Won and D. Keiswetter, "Electromagnetic induction spectroscopy," in Detection and Remediation Technologies for Mines and Mine-Like Targets III, A. C. Dubey, J. F. Harvey, and J. Broach, eds., Proc. SPIE 3392, pp. 14-22, 1998.

18. I. Won, D. Keiswetter, and E. Novikova, "Electromagnetic induction spectroscopy," Journal of Environmental and Engineering Geophysics, 3, pp. 27-40, 1998.

19. I. J. Won, D. A. Keiswetter, D. R. Hanson, E. Novikova, and T. M. Hall, "Gem-3: A monostatic broadband electromagnetic induction sensor," Jour. Environmental and Engineering Geophysics 2, pp. 53-64, 1997.

20. I. J. Won, D. A. Keiswetter, and T. H. Hall, "Electromagnetic induction spectroscopy for clearing landmines," IEEE Transactions on Geoscience and Remote Sensing 39, pp. 703-709, 2001. 